\title{
AVALIAÇÃO HEPATOTÓXICA DO EXTRATO AQUOSO DAS FOLHAS E SEMENTES DE PIMPINELLA ANISUM PROVENIENTES DO SERTÃO DA PARAIIBA
}

\author{
HEPATOTOXIC EVALUATION OF THE WATER EXTRACT OF \\ PIMPINELLA ANSIUM LEAVES AND SEEDS FROM INSIDE PARAIBA
}

Anne Carolinne da Silva Linhares ${ }^{1}$ Vanessa Erika Ferreira Abrantes Coutinho ${ }^{2}$

Fernando Magno Bitu Magalhães ${ }^{3}$ Wigna Luana de Figueirêdo Pimenta ${ }^{4}$ Otávio Soares Nascimento ${ }^{5}$ Mariana Gomes Pinto ${ }^{6}$

\begin{abstract}
RESUMO: Objetivo: Investigar o potencial hepatótoxico agudo do extrato aquoso da Pimpinella anisum, administrado por via oral, em ratos fêmeas e machos do Biotério da Faculdade Santa Maria. Método: Foram feitas análises bioquímicas, e análises nas mudanças morfológicas do fígado. Os ratos foram divididos em dois grupos, contendo 08 animais cada, um grupo controle tratado com água e o outro para a administração do extrato aquoso da Pimpinella anisum, ambos com a aplicação de distintos exames bioquímicos: AST, ALT, Bilirrubina, Fosfatase Alcalina e Gama-GT e comparados entre si. Resultados e Discussão: No estudo da hepatotoxicidade oral aguda, os animais receberam doses de $3 \mathrm{ml}$ do exatrato aquoso de Pimpinella anisum para $150 \mathrm{~g}$ de peso e foram observados durante 15 dias onde não apresentaram nenhum efeito adverso. Conclusão: Observou-se que não houve alterações no peso e na morfologia do fígado, nem nos exames bioquímicos, de acordo com metodologia empregada.
\end{abstract}

Palavras chave: Pimpinella anisum. Hepatotoxicidade. Extrato aquoso.

\footnotetext{
1 Biomédica. Pós-Graduanda Especialização em Microbiologia Clínica na Faculdade Integrada de Patos.

${ }^{2}$ Bacharela e Licenciada em Ciências Biológicas. Mestre em Biologia Celular e Molecular pela UFPB. Docente da Faculdade Santa Maria - FSM.

${ }^{3}$ Farmacêutico. Mestre em Sistemas Agroindustriais pela UFCG.

${ }^{4}$ Biomédica. Pós-Graduanda Especialização em Hematologia Clínica no Centro Universitário Leão Sampaio, Discente do Curso de Farmácia na Faculdade São Francisco da Paraíba.

${ }^{5}$ Biomédico graduado pela Faculdade Santa Maria - Cajazeiras, PB.

${ }^{6}$ Acadêmica do curso de Fisioterapia da Faculdade Santa Mara - FSM.
} 
ABSTRACT: Objective: of this research was to investigate the acute hepatotoxic potential of the aqueous extract of Pimpinella anisum, administered orally, in male and female rats of the Biotherium of Santa Maria College. Method: For this toxicity test, biochemical analyzes and analyzes on morphological changes of the liver were performed. The rats were divided in two groups, containing 08 animals each, a control group treated with water and the other for the administration of the aqueous extract of Pimpinella anisum, both with the application of different biochemical tests: AST, ALT, Bilirubin, Alkaline Phosphatase and Gama-GT and compared to each other. Results and discussion: In the study of acute oral hepatotoxicity, the animals received doses of $3 \mathrm{ml}$ of the aqueous exatrato of Pimpinella anisum to $150 \mathrm{~g}$ of weight and were observed during 15 days where they did not present any adverse effect. Conclusion: It was observed that there were no changes in liver weight and morphology, nor in biochemical tests, according to the methodology used.

Keywords: Pimpinella anisum. Hepatotoxicity. Aqueous extract. 


\section{INTRODUÇÃO}

Há milênios as plantas são utilizadas de maneira medicinal em todo o mundo para o tratamento de muitas doenças que cercam a humanidade. Existe uma vasta quantidade de plantas que são usadas pelo senso comum a fim de tratar enfermidades (DE ARAÚJO et al, 2014.) De acordo com a Organização Mundial de Saúde, $80 \%$ da população mundial fazem uso de plantas medicinais. No Brasil, essa prática foi deixada como herança pelos povos indígenas e africanos passando para as futuras gerações uma poderosa alternativa para o tratamento de doenças. (OLIVEIRA, 2013.).

São popularmente utilizadas em formas de chás, óleos essenciais, insumos, xaropes, lambedores, onde seus princípios ativos, através da farmacocinética e farmacodinâmica reagem com o organismo com o intuito de tratar problemas, principalmente nos sistemas fisiológicos, como o do trato gastrointestinal, respiratório, neurológico, inflamações, mialgias entre outras enfermidades (FIGUEIREDO, 2014).

Diante do conhecimento do senso comum o uso de plantas medicinais geralmente não oferece riscos para o organismo, visando o lado de que o que provêm da natureza pode não ser prejudicial. De acordo com Freitas, 2014, a literatura e vários outros estudos indicam que o uso indiscriminado de determinadas plantas resultou em intoxicações e em casos extremos, inclusive falência de órgãos. Estima-se que apenas cerca de apenas 15\% das 300 mil espécies de plantas foram submetidas a estudos científicos para a verificação de sua eficácia ou toxicidade (RAMALHO et al, 2015).

Nas plantas são encontradas substâncias químicas, intituladas princípios ativos, os componentes encontrados podem ser: alcalóides, glicosídeos, cardioativos, glicosídeos cianogênicos, taninos, saponinas, oxalato de cálcio e toxialbuminas, entre outros princípios que podem causar problemas celulares mutacionais, hepatotoxicidade e nefrotoxicidade isso a curto ou longo prazo, 
dependendo, das dosagens e freqüência no uso dessas plantas. (DA COSTA MENDIETA, 2014). De acordo com os últimos dados do Sistema Nacional de Informações Tóxico Farmacológicas (SINITOX), em 2013, no Brasil foram registrados 441 casos de intoxicação por plantas, sendo 35 na região Nordeste.

A Erva doce (Pimpinella anisum), também conhecida como, anis ou anisverde, é uma erva aromática da família Apiaceae encontrada no mundo todo, porém é nativa do sudoeste da Ásia e do Mediterrâneo oriental. Os seus frutos são utilizados como condimento e na produção de licores e óleos essenciais. Como planta medicinal, é geralmente usada como um antipirético, antiparasitários, antifúngicos e distúrbios digestivos, em forma de pó, de infusão, tinturas e xaropes (PINO et al, 2012).

Diante disso e do fato de existirem poucos estudos relacionados a hepatotoxicidade dos componentes fitoterápicos da Pimpinella anisum, a avaliação toxicológica é de extrema importância para a qualidade e segurança da população e das indústrias que fazem uso desse produto natural, tão utilizados a milênios em todo o mundo. Tendo em vista uma forma segura e eficiente do uso dessa planta o presente estudo teve como objetivo avaliar a hepatotoxicidade do extrato aquoso de sementes e folhas de Pimpinella anisum.

\section{MATERIAIS E MÉTODOS}

\subsection{Materiais}

A espécie Pimpinella anisum foi adquirida em uma loja de produtos naturais, localizada no município de Cajazeiras, Paraíba, Brasil. O material foi embalado e transportado até o laboratório de pesquisa em Biomedicina da Faculdade Santa Maria, em Cajazeiras-PB. 


\subsubsection{Extrato Aquoso}

Uma porção do material foi triturada, após fervida por 2 minutos em $150 \mathrm{~mL}$ água mineral, utilizando um fogão para o aquecimento. Após um minuto em repouso, o material foi filtrado com papel filtro rendendo aproximadamente $40 \mathrm{~mL}$ de extrato.

\subsubsection{Animais}

Foram utilizados 16 ratos, 08 para o grupo controle e 08 para a aplicação do extrato, albinos, fêmeas e machos, Wistar, adultos jovens, de peso entre 130-160g, provenientes do biotério da Faculdade Santa Maria, Cajazeiras-PB. Os animais foram mantidos em gaiolas de polietileno, sob condições monitoradas de temperatura $\left(20 \pm 3^{\circ} \mathrm{C}\right.$, ) ciclo claro/escuro de 12 horas, sendo a fase clara de 06 às 18 horas, recebendo ração padrão $N_{u}$ ilabß e água ad libitum disponíveis em garrafas de polietileno com bicos de inox.

\subsection{Metodologia}

\subsubsection{Condições experimentais}

Os procedimentos foram realizados no laboratório de habilidades cirúrgicas, Bioquímica e Análises Clínicas da Faculdade Santa Maria - FSM, no período compreendido das 7:00 às 12:00 horas. Todos os animais foram mantidos durante 3 horas antes do experimento na sala onde foi desenvolvido o estudo, a fim de adaptálos ao ambiente experimental. Antes de cada procedimento, as bancadas e os 
aparelhos utilizados foram higienizados com álcool a $70 \%$. A pesquisa seguiu em conformidade e foi conduzida em obediência às normas e diretrizes bioéticas vigentes para ensaios envolvendo seres vivos: animais (Guide for the Careand Use of Laboratory Animals, do NIH - National Institute of Health - EUA, 1996; Lei Federal NN 11.794/2008; Conselho Nacional de Controle de Experimentação - CONCEA); e integridade da fauna e flora (Lei Federal № 9605/1998).

\subsubsection{Avaliação da hepatotoxicidade aguda}

\subsubsection{Screening hipocrático}

Os animais foram divididos em dois grupos, sendo 08 animais por caixa. Um dos grupos recebeu a dose de $3 \mathrm{ml}$ por $150 \mathrm{~g}$ de peso do extrato aquoso da Pimpinella anisum por via oral para grupo tratado e água para grupo controle durante 15 dias. Após administração, foram observados os efeitos gerais apresentados pelos animais experimentais nos intervalos: 0, 2, 4, 8 e 24 horas no primeiro dia e uma vez ao dia, sempre no mesmo horário, nos 15 dias seguintes, com o objetivo de observar possíveis alterações comportamentais. Os parâmetros analisados foram atividade geral, resposta ao aperto de calda, convulsão, motilidade, tremores e morte. Após o $15^{\circ}$ dia, os animais foram anestesiados com uma solução de 4,5ml de Cloridrato de ketamina (C), 1,5 ml Cloridrato de Xilasina (X) e $34 \mathrm{ml}$ de solução Salina para a administração na proporção de $1 \mathrm{ml}$ para $100 \mathrm{~g}$ de peso, Intraperitoneal, colocados em decúbito dorsal para coleta do sangue através de punção cardíaca, como também retirada do fígado. O teste de toxicidade oral aguda foi realizado segundo o protocolo experimental Guideline 423 (OECD 423; 2001) seguindo a proposta da Sociedade Brasileira de Ciência em Animais de Laboratório/Colégio Brasileiro de Experimentação Animal (COBEA). 


\subsubsection{Massa relativa do fígado}

Após eutanásia dos animais, o fígado foi retirado e pesado. $\mathrm{O}$ cálculo da massa relativa do órgão de cada animal foi realizado dividindo-se o peso de cada órgão (g) pelo peso corporal de cada animal no dia da coleta, e multiplicando-se o resultado por 100 . O resultado foi expresso em g/100 g de peso vivo (g/100g p.v.).

Figura 2: Retirada do fígado para pesagem, observação da morfologia e números para obtenção da média.
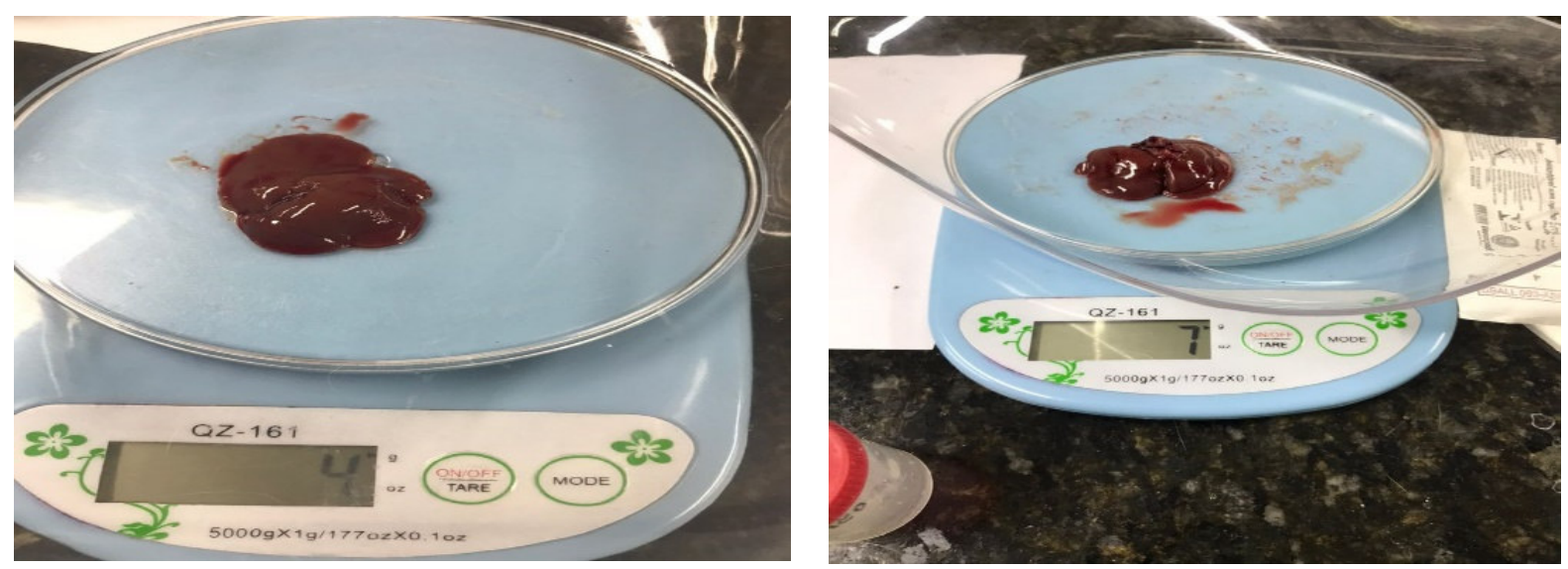

FONTE LINHARES, 2019.

\subsubsection{Análise bioquímica}

As amostras sanguíneas foram coletadas em tubos sem anticoagulante e com gel separador e ativador de coágulo para obtenção do soro. Após centrifugação, (2500 rpm 10 minutos), os parâmetros bioquímicos foram analisados pelo método cinético, tendo como exames Aspartato aminotransferase - AST - e alanina aminotransferase - ALT, Bilirrubina, Albumina, Gama - GT e Fosfatase alcalina, Os exames foram feitos em um laboratório terceirizado. 


\subsubsection{Exames bioquímicos}

As análises sanguíneas dos animais foram terceirizadas. Os ratos passaram por um jejum prévio de 5 horas, depois disso, foram anestesiados com uma solução de 4,5 ml de Cloridrato de ketamina (C), 1,5 ml Cloridrato de Xilasina (X) e $34 \mathrm{ml} \mathrm{ml}$ de solução Salina para a administração na proporção de $1 \mathrm{ml}$ para $100 \mathrm{~g}$ de peso, Intraperitoneal. A coleta do sangue foi realizada através de punção cardíaca. O soro foi obtido por centrifugação do sangue em tubos contendo gel separador, a 2500 rpm por 10 minutos. Em seguida enviados para o laboratório para a realização dos seguintes exames bioquimicos: aspartato transaminase (AST), alanina transaminase (ALT), Bilirrubina, albumina, Gama-GT e fosfatase alcalina.

Figura 3: Rato anestesiado, para a realização da pulsão cardíaca; exposição dos órgãos e retirada do fígado.

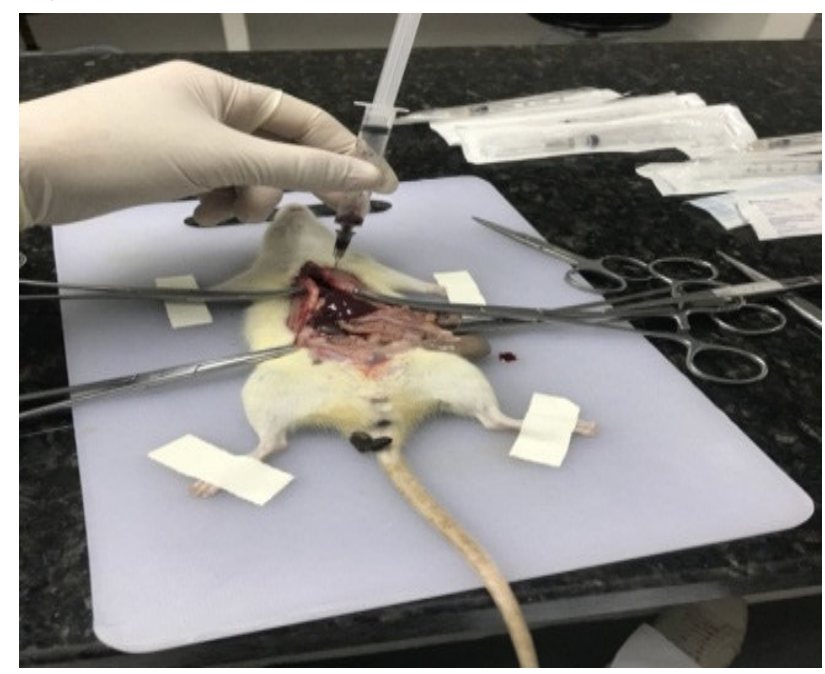

Fonte: Linhares, 2019. 


\section{RESULTADOS E DISCUSSÕES}

As plantas apresentam alguma toxicidade dependendo da dosagem, entretanto a denominação de plantas tóxicas se aplica a todos os vegetais que, através da ingestão, inalação ou contato, causam danos à saúde, isso tanto para o ser humano quanto para animais, até casos extremos, levando à morte. Nessas plantas podem ser encontrados componentes que podem desenvolver diferentes reações ou tornarem-se perigosas devido à coleta ou uso inadequado dos constituintes e até mesmo na forma que é preparada. (DA COSTA MENDIETA, 2014). O uso de plantas para fins medicinais é muito utilizado por diversas classes sociais. Evidenciando a importância destes aspectos, este estudo foi realizado, objetivando a investigação da toxicidade aguda do Extrato aquoso da Pimpinella anisum.

O peso e os resultados obtidos nos exames bioquimicos através do soro dos ratos, caracterizados pelos principais marcadores hepáticos, (Bilirrubina Direta, Bilirrubina Total, Gama - Gt, Fosfatase Alcalina, AST e ALT.), foram expressos nas tabelas abaixo:

Após a eutanásia houve pulsão cardíaca e a retirada do fígado para a pesagem, morfologicamente não apresentou nenhuma alteração, nem diferença significativa na comparação das médias obtidas entre o peso dos fígados dos dois grupos testados.

Tabela 01: Massa relativa do órgão (fígado), após eutanásia dos ratos tratados com água e grupo tratado com o extrato aquoso da Pimpinella anisum.

\begin{tabular}{lcc}
\hline & Orgão & Peso do orgão Média \\
\hline Grupo Controle & Fígado & $\mathbf{5 , 3 g}$ \\
Grupo Pimpinella anisum & Fígado & $\mathbf{5 , 7 g}$ \\
\hline
\end{tabular}

Fonte: Linhares, 2017. 
Os ratos foram divididos em dois grupos, contendo 08 animais cada, um grupo controle e o outro para a administração do extrato aquoso, ambos com a aplicação de distintos exames bioquímicos: AST, ALT, Bilirrubina, Fosfatase Alcalina e Gama-GT e comparados entre si.

Tabela 02: Resultados dos exames bioquímicos, dos ratos tratados com água. (Expresso os exames de cada animal). Onde: Grupo Controle - C.T.n (animal tratado com água), BD (Bilirrubina direta), BT (Bilirrubina total), GGT (Gama-Gt), FALS (Fosfatase Alcalina), AST (Aspartato aminotransferase), ALT (Alanina aminotransferase).

\begin{tabular}{lcccccc}
\hline Controle Padrão & BD & BT & GGT & FALS & AST & ALT \\
\hline C.T.1 & 0,1 & 0,2 & 70 & 221 & 143 & 54 \\
C.T.2 & 0,1 & 0,2 & 50 & 332 & 199 & 79 \\
C.T.3 & 0,1 & 0,2 & 55 & 329 & 161 & 66 \\
C.T.4 & 0,1 & 0,2 & 62 & 300 & 143 & 60 \\
C.T.5 & 0,1 & 0,2 & 53 & 313 & 153 & 65 \\
C.T.6 & 0,1 & 0,2 & 54 & 196 & 170 & 62 \\
C.T.7 & 0,1 & 0,2 & 70 & 182 & 355 & 75 \\
C.T.8 & 0,1 & 0,2 & 80 & 214 & 163 & 54 \\
\hline
\end{tabular}

Fonte: Linhares, 2017.

Tabela 03: Resultados dos exames bioquímicos, dos ratos tratados com extrato da Pimpinella anisum. (Expresso os exames de cada animal). Onde: Pimpinella anisum - P.A.n (animal tratado com o extrato da Pimpinella anisum), BD (Bilirrubina direta), BT (Bilirrubina total), GGT (Gama-Gt), FALS (Fosfatase Alcalina), AST (Aspartato aminotransferase), ALT (Alanina aminotransferase).

\begin{tabular}{lcccccc}
\hline Pimpinella anisum & BD & BT & GGT & FALS & AST & ALT \\
\hline P.A. 1 & 0,1 & 0,2 & 70 & 127 & 141 & 48 \\
P.A. 2 & 0,1 & 0,2 & 50 & 301 & 156 & 77 \\
P.A. 3 & 0,1 & 0,2 & 80 & 385 & 135 & 71 \\
P.A. 4 & 0,1 & 0,2 & 80 & 374 & 298 & 81 \\
P.A. 5 & 0,1 & 0,2 & 70 & 127 & 141 & 49 \\
P.A. 6 & 0,1 & 0,2 & 90 & 334 & 257 & 78 \\
P.A. 7 & 0,1 & 0,2 & 114 & 205 & 93 & 60 \\
P.A. 8 & 0,1 & 0,2 & 50 & 184 & 300 & 62 \\
\hline
\end{tabular}

Fonte: Linhares, 2017. 
A variância comparativa dos resultados obtidos acerca dos 05 exames bioquímicos foi dada através da média, desvio padrão e o test $t$ de Student, onde o último foi quem definiu a relevância dos resultados, diante disso e da comparação entre tabelas, a planta não apresentou hepatotoxicidade, tendo em vista a metodologia aplicada, o tempo de administração e os resultados obtidos com o teste estatístico, a hipótese nula não pôde ser rejeitada.

Tabela 04: Parâmetros obtidos dos exames bioquímicos do soro de ratos tratados com água. Dados expressos como (Média e Desvio Padrão).

\begin{tabular}{lcccccc}
\hline Grupo controle & & & & & & \\
\hline & BD & BT & GGT & FALS & AST & ALT \\
Média & 0,1 & 0,2 & 61,7 & 260,8 & 185,8 & 64,3 \\
Desvio Padrão & 1,48 & 2,96 & 10,62 & 63,43 & 70,63 & 9,022 \\
\hline
\end{tabular}

Fonte: Linhares, 2017

Tabela 05: Parâmetros obtidos dos exames bioquímicos do soro de ratos tratados com o

extrato da Pimpinella anisum. Dados expressos como (Média e Desvio Padrão).

\begin{tabular}{lcccccc}
\hline \multicolumn{2}{l}{ Grupo Pimpinella anisum } & & & & & \\
\hline & BD & BT & GGT & FALS & AST & ALT \\
Média & 0,1 & 0,2 & 75,5 & 254,6 & 190,1 & 65,7 \\
Desvio Padrão & 1,48 & 2,96 & 21,02 & 106,72 & 81,63 & 12,98 \\
\hline
\end{tabular}

Fonte: Linhares, 2017.

Apesar disso a erva ainda precisa de mais alguns estudos, pois ela não apresentou hepatotoxicidade diante das estatísticas, e esses resultados poderiam hipoteticamente variar de acordo com o tempo de administração e quantidade da aplicação do extrato aquoso, visto que o Gama GT apresentou um leve aumento no seu valor $(p=0,06)$.

Tabela 06: Parâmetros obtidos através do teste de hipóteses - teste $t$ - para comparação entre os resultados obtidos nas tabelas de exames bioquimicos. 


\begin{tabular}{cccccc}
\hline BD & BT & GGT & FALS & TGO & TGP \\
\hline $\mathbf{X}$ & $\mathrm{X}$ & 0,06 & 0,4 & 0,4 & 0,4 \\
\hline
\end{tabular}

*valores referentes ao valor $\mathrm{P}$ unicaudal $\mathrm{P}(\mathrm{T}<=\mathrm{t})$ uni-caudal $\times \mathrm{p}<0,05$ (teste $\mathrm{t}$ de Student) no programa EXCEL 2016; Fonte: Linhares, 2017.

Durante 15 dias tanto o grupo controle como o grupo tratado foi submetido ao mesmo estresse, observados, e não apresentaram nenhuma alteração no comportamento ou qualquer efeito adverso.

Em outra pesquisa em que o chá de Ayahuasca foi administrado em ratos, no qual foi empregada uma metodologia semelhante à desse estudo, diante dos resultados das análises bioquímicas, não houve evidências de lesão hepática, pois não houve aumento dos parâmetros AST, ALT, Gama GT, Fosfatase alcalina. Entretanto, ocorreu redução estatística na comparação dos níveis de Fosfatase alcalina e Gama Gt em U/L entre os grupos controle e tratado. Gama GT (MannWhitney Test $=0,008$ ) e da Fosfatase Alcalina (Mann-Whitney Test $=0,0232$ ). Esses resultados podem ter alguma relação como a redução do hepatócito e do núcleo observada como alteração histológica, por intermédio de problemas em algum processo de síntese dessas enzimas, que pode ser na transcrição ou em outro local da síntese hepática das mesmas. (ACHAREZZI et al, 2015).

Existem estudos comprobatórios realizados com o extrato aquoso de $A$. sativum (alho) em camundongos Swiss machos, com a administração única por via oral de 2, 4, 8, 16 e $32 \mathrm{~g} / \mathrm{kg}$ para avaliar a toxicidade aguda, não foi examinado mortes e os ratos demonstraram uma diminuição de estímulos a ruídos e perdas no peso corporal. Entretanto, no mesmo estudo realizado em ratos Wistar (machos e fêmeas), com a administração do extrato aquoso de $A$. sativum durante 30 dias para avaliar a toxicidade subaguda ou crônica, os resultados apresentaram aumento da concentração sérica da enzima AST, apesar de não ser específico, indica possível lesão hepática, porém também pode estar elevado em situações de ataque cardíaco ou lesões musculares. Em relação ao grupo controle houve o aumento do peso do fígado, rins e coração. (FERNANDES, 2016).

Até vegetais muito conhecidos e consumidos podem apresentar toxicidade, como é o caso da batata inglesa (Solanum tuberosum $L$ ), que possui substâncias 
como os glicoalcalóides, que são tóxicos, e que podem causar reações adversas como diarréia, dor abdominal e vômito. Porém, geralmente não se associa os sintomas apresentados ao consumo desse vegetal. (CAMPOS et al, 2016). Visto que são plantas e vegetais constantemente usados no dia-a-dia, assim como o consumo da erva-doce (Pimpinella anisum).

Os principais constituintes do óleo essencial da Pimpinella anisum são $90 \%$ anetol, 2-4\% gama-P-anisaldeído, 0,9-1,5\% de metil-chavicol, 3\% de cis-pseudoisogenil-2-Metilbutirato de metilo e 1,3\% de 2-metilbutirato de trans-pseudoisoeguenilo. Estudos comprobatórios sobre o óleo essencial de P.anisum confirmaram que o mesmo não foi eficaz contra carbono hepatotoxicidade aguda induzida por tetracloreto em ratos. (CENGIZ, 2008).

\section{CONCLUSÃO}

De acordo com a metodologia, tempo de administração e dosagem, não ocorreu hepatotoxicidade, nem alteração morfológica. Observou-se que não houve aumento significativo nos parâmetros bioquímicos do grupo tratado. 


\section{REFERÊNCIAS BIBLIOGRÁFICAS}

ACHAREZZI, Bruno et al. Avaliação hepática em ratos Wistar expostos Ao chá de Ayahuasca. Saúde e Pesquisa, v. 8, n. 3, p. 423-430, 2015.

CAMPOS, S. C. et al. Toxicidade de espécies vegetais. Rev. bras. plantas med, v. 18, n. 1 , supl. 1, p. 373-382, 2016.

CENGIZ, Nureddin; OZBEK, H.; HIM, Aydın. Hepatoprotective effects of Pimpinella anisum seed extract in rats. Pharmacologyonline, v. 3, p. 870-874, 2008.

DA COSTA MENDIETA, Marjoriê et al. Plantas tóxicas: importância do conhecimento para realização da educação em saúde. Revista de enfermagem UFPE on line-ISSN: 1981-8963, v. 8, n. 3, p. 680-686, 2014.

DE ARAÚJO, Ferreira et al. Perfil e prevalência de uso de plantas medicinais em uma unidade básica de saúde da família em Campina Grande, Paraíba, Brasil. Revista de Ciências Farmacêuticas Básica e Aplicada, v. 35, n. 2, 2014.

FIGUEIREDO, Djailton Ramos de. Avaliação da citotoxicidade do extrato hídrico da erva doce (Pimpinella anisum L.) através do teste em Allium. cepa L. 2014.

NOBRE, Márcia. Toxicidade dos fitoterápicos de interesse do SUS: uma revisão. Semina: Ciências Biológicas e da Saúde, v. 37, n. 1, p. 91-104, 2016.

OLIVEIRA, Roseli F. et al. Evaluation of the hepatotoxicity of Abarema cochliacarpos extracts in mice Mus musculus. Revista Brasileira de Farmacognosia, v. 23, n. 4, p. 674-679, 2013.

PINO, Oriela et al. Composición química y actividad antibacteriana del aceite esencial de Pimpinella anisum. L. Revista de Protección Vegetal, v. 27, n. 3, p. 181-187, 2012.

RAMALHO, Josue Amaral et al. Toxicidade Aguda em Ratos Wistar Tratados com o Extrato Etanólico de Dioclea grandiflora Mart. Ex Benth (Fabaceae) (EEDg). Revista Brasileira de Ciências da Saúde, v. 18, n. 4, p. 279-286, 2015. 